\title{
MATERIAL SYMMETRY AND THE EVOLUTION OF ANISOTROPIES IN A SIMPLE MATERIAL-I. CHANGE OF REFERENCE CONFIGURATION
}

\author{
Mehrdad Negahban* and alan S. Wineman \\ Department of Mechanical Engineering and Applied Mechanics. The University of Michigan, \\ Ann Arbor, MI 48109, U.S.A.
}

(Receited 18 October 1988; in revised form 10 March 1989)

\begin{abstract}
Noll's rule is used to determine the structure of a material symmetry group written with respect to one reference configuration when the representation of the symmetry with respect to another configuration is the traditional material symmetry group associated with isotropy, transverse isotropy or orthotropy, and for an arbitrary deformation gradient relating the two configurations. It is shown that the former symmetry group can contain an orthogonal subgroup. It is determined whether this subgroup is that for isotropic, transversely isotropic. orthotropic, monoclinic, or triclinic response. and the preferred directions of the symmetry are determined.
\end{abstract}

\section{INTRODUCTION}

In the theory of constitutive equations, a material's symmetry properties are described by specifying a reference configuration and a set of linear transformations which map the reference configuration onto mechanically equivalent configurations. This set of transformations forms a material symmetry group. If the reference configuration is changed, there will be a different material symmetry group representing the same material symmetry. Noll [1] has presented a rule which expresses the second material symmetry group in terms of the first and in terms of the deformation gradient of the mapping between the two reference configurations.

Though Noll's rule has long been available, it has not been gainfully exploited in determining the properties of the second material symmetry group. For instance, consider a material whose symmetry group with respect to some configuration is the full orthogonal group, i.e. that for isotropy. Let a new reference configuration be introduced by a mapping corresponding to that of uniaxial extension. It is commonly assumed that the material symmetry group for this new reference configuration is that for transverse isotropy. This common perception is incorrect. An application of Noll's rule shows that the new symmetry group contains those orthogonal transformations which describe transverse isotropy, and also unimodular non-orthogonal transformations. A detailed discussion of this case, as well as the corresponding implications for representations of constitutive equations has been given by Wineman et al. [2].

In the present work, we use Noll's rule to determine the structure of the material symmetry group associated with a new reference configuration when the original material symmetry group is that for isotropy, transverse isotropy or orthotropy, and for an arbitrary deformation gradient relating the two configurations. In particular, we show that the new symmetry group contains an orthogonal subgroup, determine whether it is that for isotropic, transversely isotropic, orthotropic, monoclinic, or triclinic response, and determine the preferred directions of the symmetry.

Fundamental remarks and terminology are introduced in Section 2, Noll's rule in Section 3. A property of material symmetry groups is discussed in Section 4. In Section 5 we introduce the notion of the apparent material symmetry group, i.e. the orthogonal subgroup. In Section 6 we establish a property satisfied by the transformations of this apparent material symmetry group. This property is exploited by the use of anisotropic tensors. which are reviewed in Section 7. We also present symmetry transformations and anisotropic tensors for triclinic, monoclinic, orthotropic, and transversely isotropic materials. In Section 8, a method is established for solving for the transformations which satisfy the \footnotetext{
0347.

* Present address: Department of Engineering Mechanics, University of Nebraska-Lincoln, Lincoln, NE 68588 .
} Contributed by K. R. Rajagopal. 
above-mentioned property. These results are applied, in Sections 9-11, to the determination of the apparent material symmetry groups for isotropic materials, transversely isotropic materials, and orthotropic materiais, respectively.

\section{FUNDAMENTAL REMARKS AND TERMINOLOGY}

This section is devoted to the development of the idea of material symmetry (MS), which is a statement on the mechanical equivalence of neighborhoods (nbhds) of a material point.

The nbhd of influence of a particle $X$ is an ordered set which contains all material points which directly influence the response at particle $X$. We will denote the nbhd of influence of particle $\mathrm{X}$ in configuration $\kappa$ by $\mathscr{N}_{\kappa}(\mathrm{X}), \mathscr{V}_{\kappa}(\mathrm{X})$ is ordered by how these material points appear in configuration $\kappa$. Figure 1 shows two representations of the nbhd of influence of particle $X$.

For a simple material, ${ }^{\dagger}$ the nbhd of influence is defined to be the smallest nbhd of the particle under consideration. From this point on we will use the term nbhd when we actually mean the nbhd of influence in a simple material. We shall also cease all reference to the particular material point under consideration, although there is always a single material point under consideration.

An MS represents a reorganization of the nbhd which leaves the reorganized nbhd mechanically indistinguishable from the original nbhd. MS can be seen in a crystalline material where a well-defined symmetry exists between the arrangement of the molecules on a molecular level, or it can be seen in an amorphous polymer on a macromolecular leveleven without a molecular level structure-due to the random orientation of long-chain molecules. In a continuum model, we are strictly concerned with the macroscopic response of the material and we will define MS strictly in regards to this macroscopic response; that is, an MS represents a reorganization of the nbhd which leaves the reorganized nbhd indistinguishable from the original nbhd in its macroscopic response to all possible histories of events. We will consider these two nbhds - the original nbhd and its reorganization-to be two materially equivalent nbhds. This definition of MS allows the consideration of all different levels of symmetry-from the microscopic to the macroscopic-without ignoring the influence of interaction between different levels of structure-or lack of structure-in the actual material.

To represent an MS, one needs to have at least one representation of the nbhd under consideration which will be used as reference, and a linear transformation which takes this
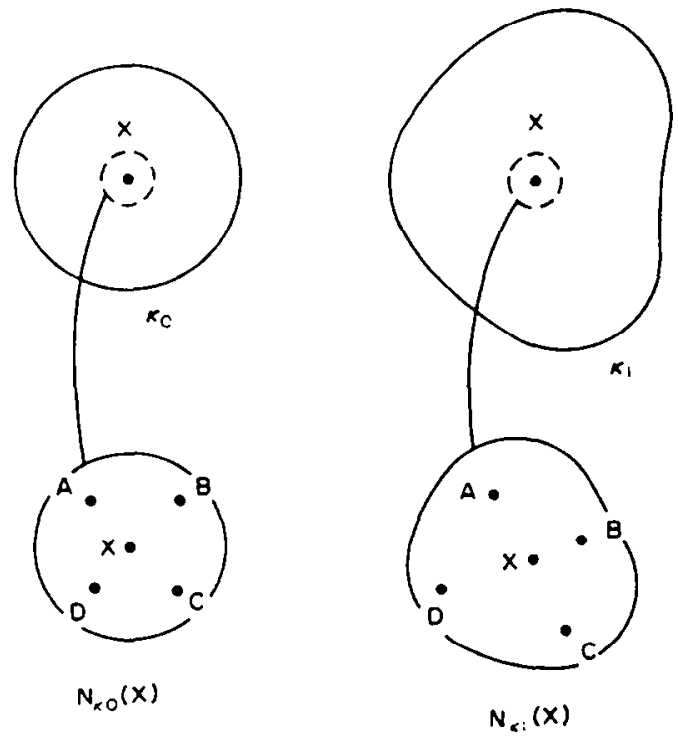

Fig. 1. A single nbhd of influence of particle $X$ represented with respect to two different configurations $\kappa_{0}$ and $\kappa_{1}, A, B, C$, and $D$ are four particles of the nbhd of influence of $X$.

A material whose response at any material point can be modeled by a functional of the history of the first deformation gradient at that point. 

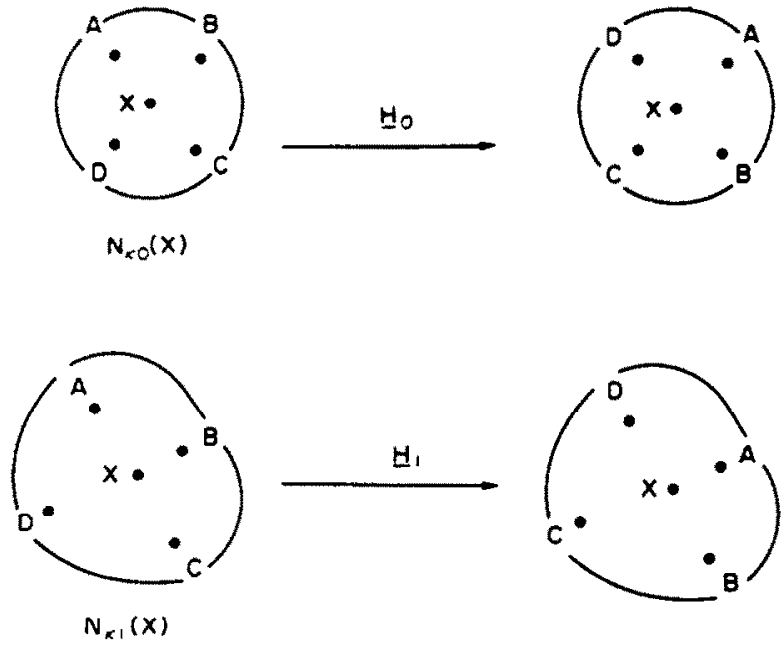

Fig. 2. The same reorganization represented with respect to two different configurations $\kappa_{0}$ and $\kappa_{1}$ taken as reference. $H_{0}$ is the transformation representing the $M S$ with respect to $\kappa_{0}$ and $H_{1}$ is the transformation representing the same MS with respect to $\kappa_{1}, A, B, C$, and D are four particles of the nbhd of influence of $X$.

representation into a materially equivalent representation. In Fig. 2 we show two nbhds of particle $X$ and how the same $M S$ is represented by different transformations $H_{0}$ and $H_{1}$, depending on the choice of the configuration used as reference for the representation of the MS. This MS allows the replacement of particle A by D, B by A, C by B, and D by C.

To avoid repetition, we assume all linear transformations to be non-singular (in the sense that their determinants are not equal to zero).

All members of an MS group must be unimodular (volume preserving) transformations. We have introduced the notion of representing an MS by a linear transformation $\mathbf{H}_{0}$ of a pre-selected reference nbhd represented by $\mathscr{N}_{x_{0}}(\mathrm{X})$ (see Fig. 2). The transformation $\mathbf{H}_{0}$ represented a reorganization of the nbhd of particle $X$. That is, $H_{0}$ replaces each member of $\hat{H}_{\mathrm{x}_{0}}$ by either itself or another member of $\mathscr{N}_{\mathrm{x}_{0}}$. As each material point in $\mathcal{N}_{\mathrm{k}_{0}}$ is represented by its location in the configuration $\kappa_{0}, \mathrm{H}_{0}$ is a one-to-one mapping of the space occupied by $\mathcal{A}_{x_{0}}$ onto itself. This can only happen if $\mathbf{H}_{0}$ preserves volume. ${ }^{\dagger}$

\section{NOLL'S RULE}

Noll [1] has described the relation between any two transformations which are descriptions of the same MS with respect to two different configurations. The following is a presentation of this rule.

We have already shown that, given two different representations $\mathcal{N}_{\mathrm{K}_{0}}(\mathrm{X})$ and $\mathcal{N}_{\mathrm{x}_{1}}(\mathrm{X})$ of a nbhd, the same MS is represented by two different transformations $H_{0}$ and $H_{1}$ (see Fig. 2). Figure 3 shows the nbhd of particle $X$ represented in two different configurations $\kappa_{0}$ and $\kappa_{1}$. $\bar{F}$ is the deformation gradient which compares the nbhd of $X$ in configuration $\kappa_{1}$ to its configuration in $\kappa_{0} . H_{0}$ is the MS transformation which takes $\mathscr{N}_{\kappa_{0}}$ to the materially equivalent nbhd $\mathscr{N}_{\kappa_{0}^{*}} . H_{1}$ is the MS transformation which which takes $\mathscr{N}_{\boldsymbol{x}_{1}}$ to the materially equivalent nbhd $\mathscr{N}_{k_{i}^{*}}$. In Fig. 3, the transformations $\mathbf{H}_{0}$ and $\mathbf{H}_{1}$ are mappings of the nbhd of $\mathrm{X}$ onto itself, and therefore $\mathcal{N}_{x_{0}}$ actually overlaps $\mathscr{N}_{\alpha_{0}^{*}}$ and $\mathscr{N}_{x_{1}}$ actually overlaps $\mathcal{N}_{\kappa_{i}^{*}}$.

We will show that

$$
\mathbf{H}_{1}=\overline{\mathbf{F}} \mathbf{H}_{0} \overline{\mathbf{F}}^{-1} \text {. }
$$

Consider particles $\mathrm{P}$ and $\mathrm{Q}$ in the nbhd of $\mathrm{X}$. In $\mathscr{N}_{\mathrm{x}_{0}}$, the positions of $\mathrm{P}$ and $\mathrm{Q}$ with respect to the position of $X$ are given by the vectors $d p$ and dq respectively. If the MS transformation $H_{0}$ represents a reorganization which replaces particle $Q$ by $P$, then $H_{0}$ must

\footnotetext{
${ }^{\dagger}$ Requiring $\mathrm{H}_{0}$ to map the space occupied by $\mathcal{N}_{\mathrm{k} 0}$ onto itself might have other implications which are out of the scope of this work.
} 


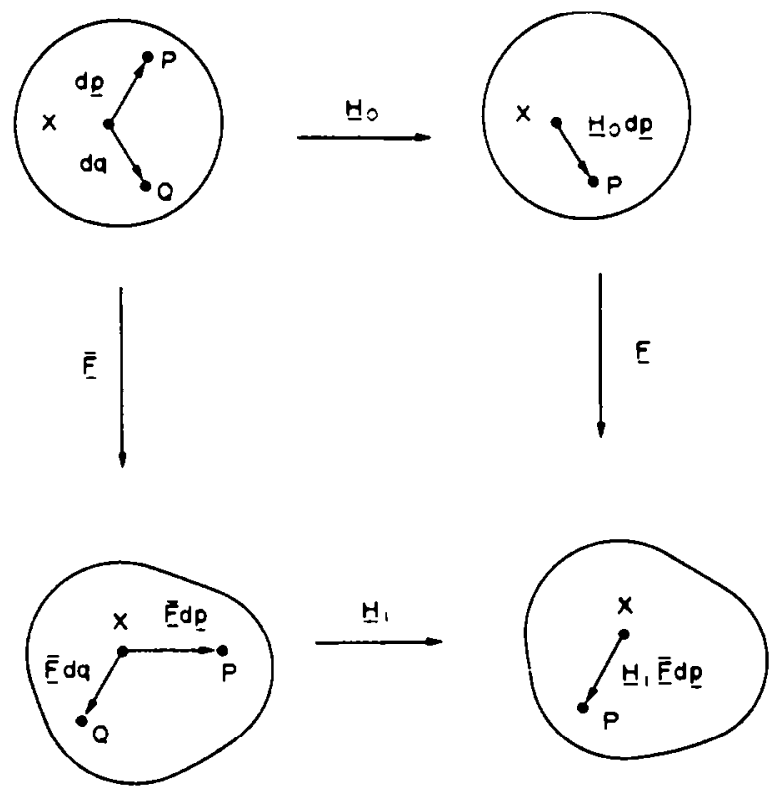

Fig. 3. Relation between two representations $H_{0}$ and $H_{1}$ of the same MS.

map $\mathrm{P}$ onto $\mathrm{Q}$ which means $\mathbf{H}_{0} \mathrm{dp}=\mathrm{dq}$. In $\mathscr{N}_{\boldsymbol{x}_{1}}$, the positions of particles $\mathrm{P}$ and $\mathrm{Q}$ with respect to the positions of $X$ are given by the vectors $\bar{F} d p$ and $\bar{F} d q$ respectively. If $H_{1}$ is supposed to represent the same reorganization as $H_{0}$ represents, then $H_{1}$ should map $P$ onto $Q$ which means that $H_{1} \bar{F} d p=\bar{F} d q$; but, as $H_{0} d p=d q$, we will have $H_{1} \bar{F} d p=\bar{F} d q$ $=\overline{\mathrm{F}} \mathbf{H}_{0} \mathrm{dp}$ which, considering that this relation should hold for all vectors $\mathrm{dp}$, yields equation (1).

The set of all transformations representing the material's different symmetries with respect to $\mathcal{N}_{\boldsymbol{k}_{0}}$ forms a mathematical group. We will call this group the material symmetry group (MSG) with respect to $\mathcal{N}_{\alpha_{0}}$ and denote it by $g_{\mathrm{k}_{0}}$. The same group of symmetries represented with respect to $\mathscr{N}_{\alpha_{1}}$ will be denoted by $g_{\alpha_{1}}$. As (1) is true for every two MS transformations $\mathbf{H}_{0} \in g_{\kappa_{0}}$ and $H_{1} \in g_{\kappa_{1}}$ which describe the same MS, (1) defines a one-to-one mapping of $g_{x_{0}}$ onto $g_{\kappa_{1}}$. This mapping was introduced by Noll [1] and will be referred to as Noll's rule. Remark 1 is a restatement of this rule.

Remark 1 (Noll's rule). Given two configurations $\kappa_{0}$ and $\kappa_{1}$ of a material body, the two MSGs $g_{\kappa_{0}}$ and $g_{\kappa_{1}}$ are related by

$$
g_{\kappa_{1}}=\overline{\mathbf{F}} g_{\kappa_{0}} \overline{\mathbf{F}}^{-1},
$$

where $\overline{\mathbf{F}}$ is the deformation gradient comparing, $\mathcal{N}_{x_{1}}$ to $\mathscr{N}_{\mathrm{x}_{0}}$.

\section{THE GROUP OF MATERIAL SYMMETRY GROUPS}

Up to this point we have assumed the existence of a set of reorganizations which describes a material's symmetries, and that $g_{\kappa_{0}}, g_{k_{1}}, g_{k_{2}}, \ldots$, are different explicit representations of this set. Let $\mathscr{G}$ be the set containing all these explicit representations of a material's symmetries. That is,

$$
\mathscr{G}=\left\{g_{\kappa_{0}}, g_{k_{1}}, g_{\kappa_{2}}, \ldots\right\} \text {. }
$$

It is simple to show that $\mathscr{G}$ is a mathematical group (see [3]). $\mathscr{G}$ will be called the group of MSGs (representing a particular MS). This group has the property that each member of it can be derived from every other member by a transformation of the form (2). This allows us to select one member of $\mathscr{G}$ as a representative member, and to generate all other members by transformations of the form (2). This is reflected in Remark 2 as follows. 
Remark 2. The group of MSGs, $\mathscr{S}$, can always be generated from any one of its members. Given any member $g_{x}, \mathscr{S}$ will be given as

$$
\mathscr{G}=\left\{g \mid g=\overline{\mathbf{F}} g_{\mathbf{x}} \overline{\mathbf{F}}^{-1} \text { for every } \overline{\mathbf{F}}\right\},
$$

where $\overline{\mathrm{F}}$ is any arbitrary deformation gradient. We will call $g_{k}$, in (4), the generator of 9 .

\section{APPARENT MATERIAL SYMMETRY GROUPS}

Next, we will look at the nature of the individual members of each MSG. As we have stated before, the members of each MSG are unimodular (volume preserving) transformations. Some of these members can be orthogonal and others might be unimodular but nonorthogonal. It is important to note that, even though the generator of $\mathscr{S}$ might contain only orthogonal members, other members of $\mathscr{G}$ will probably contain both orthogonal and unimodular non-orthogonal members. The reason for this is that the transformation given in (1) in general does not preserve orthogonality (see [2] for examples).

We will give particular importance to the orthogonal members of the MSG. There are two reasons for this special consideration. First, the orthogonal members are equivalent to central inversions and rigid body rotations, and therefore are both mathematically and experimentally simpler to work with. Second, we will show that these members are responsible for the establishment of a certain type of structure on all MSGs.

Given a MSG, we will define its associated apparent MSG to be its subset of orthogonal members. Therefore, if $\mathscr{A}_{\kappa}$ is the apparent MSG of $g_{\kappa}$, then

$$
\mathscr{A}_{x}=\left\{\mathbf{H} \mid \mathbf{H} \in g_{x} \text { and } \mathbf{H} \in \mathcal{O}\right\} \text {, }
$$

where $\mathcal{O}$ denotes the full group of orthogonal transformations. It is simple to show that $\mathscr{A}_{x}$ is a mathematical group.

\section{THE IMAGE OF AN APPARENT MATERIAL SYMMETRY GROUP AND ITS PROPERTIES}

Next, we will show how the apparent MSG of one MSG imposes a structure on other MSGs in $\mathscr{G}$. Let us say that we are given the apparent MSG $\mathscr{A}_{\mathrm{x}_{0}}$, associated with the MSG $g_{x_{0}}$. Let $\mathscr{A}_{x_{1}}\left(\mathscr{A}_{x_{0}}\right)$ be the image of $\mathscr{A}_{\kappa_{0}}$ on $g_{x_{1}}$. That is,

$$
\mathscr{B}_{\kappa_{1}}\left(\mathscr{A}_{\kappa_{0}}\right)=\left\{\mathbf{H}_{1} \mid \mathbf{H}_{1}=\overline{\mathbf{F}} \mathbf{H}_{0} \overline{\mathrm{F}}^{-1} \text { for every } \mathbf{H}_{0} \in \mathscr{A}_{\kappa_{0}}\right\} \text {, }
$$

which can also be written as

$$
\mathscr{B}_{\mathrm{x}_{\mathrm{i}}}\left(\mathscr{A}_{\mathrm{x}_{0}}\right)=\overline{\mathrm{F}} \mathscr{A}_{\mathrm{x}_{0}} \overline{\mathrm{F}}^{-1} \text {, }
$$

where $\overline{\mathbf{F}}$ is the deformation gradient comparing $\mathcal{N}_{\mathrm{k}_{1}}$ with $\mathscr{N}_{\mathrm{x}_{0}}$ (see Fig. 4). We intend to show that all members of $\mathscr{A}_{x_{1}}\left(\mathscr{A}_{\kappa_{0}}\right)$ have an exclusive common property. This will then allow us to say that there is a structure imposed on $g_{k_{1}}$ by the apparent MSG $g_{x_{0} \text {. The }}$ common property of all members of $\mathscr{X}_{\kappa_{1}}\left(\mathscr{A}_{\mathrm{K}_{0}}\right)$ comes from the fact that these members are images of orthogonal members. It is important to remind the reader that the transformation (1) does not, in general, preserve orthogonality and therefore the members of $\mathscr{P}_{k_{1}}\left(\mathscr{A}_{k_{0}}\right)$ need not be orthogonal. Every $H_{1} \in \mathscr{B}_{x_{1}}\left(\mathscr{A}_{k_{0}}\right)$ is the image of some $H_{0} \in \mathscr{A}_{\kappa_{0}}$, which has the property given by

$$
\mathbf{H}_{0} \mathbf{H}_{0}^{T}=\mathbf{H}_{0}^{T} \mathbf{H}_{0}=\mathbf{I} \text {. }
$$

Substitution of $\mathrm{H}_{0}$ by $\mathrm{H}_{0}=\overline{\mathrm{F}}^{-1} \mathrm{H}_{1} \overline{\mathrm{F}}$ in (8) from Noll's rule yields the following remark (see [3]).

Remark 3. If $\overline{\mathrm{B}}=\overline{\mathrm{F}}^{\mathrm{T}}$ for $\overline{\mathrm{F}}$ given as the deformation gradient comparing $\mathcal{N}_{\mathrm{x}_{1}}$ with $\mathscr{V}_{\mathrm{x}_{0}}$, then

$$
\mathscr{B}_{x_{1}}\left(\mathscr{A}_{x_{0}}\right)=\left\{\mathbf{H}_{1} \mid \mathbf{H}_{1} \in g_{x_{1}} \text { and } \mathbf{H}_{1} \overline{\mathbf{B}} \mathbf{H}_{1}^{T}=\overline{\mathbf{B}}\right\} \text {. }
$$

Similarly, if we introduce the apparent MSG of $g_{k_{1}}$ and its image in $g_{\alpha_{0}}$ as $\mathscr{A}_{k_{1}}$ and $\mathscr{B}_{x_{0}}\left(\mathscr{A}_{k_{1}}\right)$, respectively, then we can also show the following. 

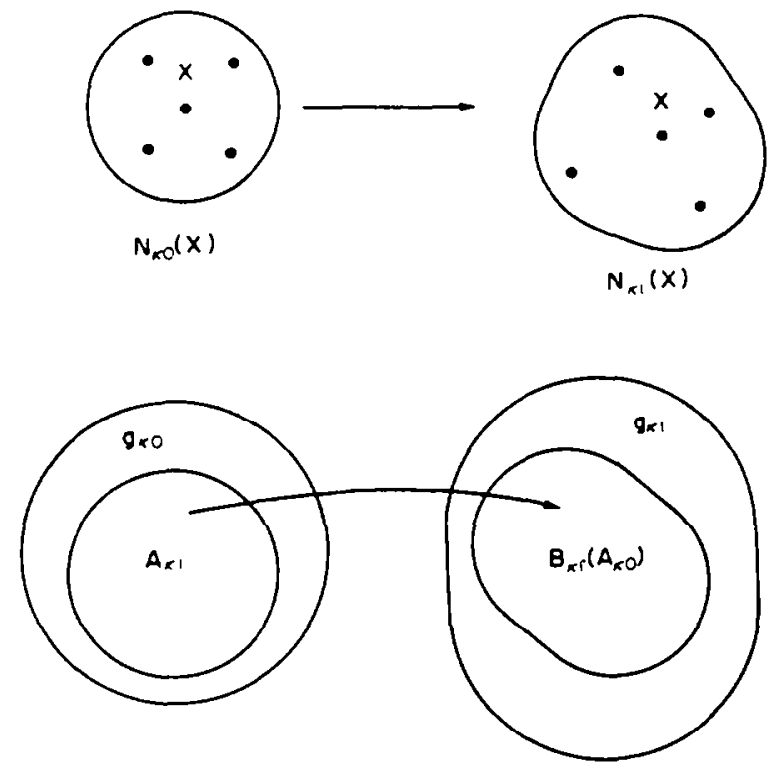

Fig. 4. The image of $\mathscr{\alpha}_{x_{0}}$ on $g_{x_{1}}$.

Remark 4. If $\overline{\mathbf{C}}=\overline{\mathbf{F}}^{T} \tilde{\mathbf{F}}$ for $\overline{\mathbf{F}}$ given as the deformation gradient comparing $\mathscr{V}_{\alpha_{1}}$ with $\mathcal{N}_{x_{0}}$, then

$$
\mathscr{B}_{\kappa_{0}}\left(\mathscr{A}_{\kappa_{1}}\right)=\left\{\mathbf{H}_{0} \mid \mathbf{H}_{0} \in g_{\kappa_{0}} \text { and } \mathbf{H}_{0}^{T} \overline{\mathbf{C}} \mathbf{H}_{0}=\overline{\mathbf{C}}\right\} \text {. }
$$

The selections of $\kappa_{0}$ and $\kappa_{1}$ in the preceding discussion were totally arbitrary. Let us select $\kappa_{1}$ to be a fixed configuration and $\kappa_{0}$ as any other configuration. Equation (9) shows that the apparent MSG of $g_{\kappa_{0}}$ imposes a structure on $g_{k_{1}}$ [i.e. each member of $\mathscr{B}_{\mathrm{k}_{1}}\left(\mathscr{A}_{\mathrm{k}_{0}}\right)$ has the exclusive property that $\left.\mathbf{H}_{1} \overline{\mathbf{B}} \mathbf{H}_{1}^{T}=\overline{\mathbf{B}}\right]$. As $\kappa_{0}$ is an arbitrary configuration, this states that the apparent MSG of each member in $\mathscr{G}$ separates $g_{\kappa,}$ into two subsets: those members which have the property $\mathbf{H}_{1} \overline{\mathbf{B}} \mathbf{H}_{1}^{T}=\overline{\mathbf{B}}$, for the given $\overline{\mathbf{B}}=\overline{\mathbf{F}} \overline{\mathbf{F}}^{T}$, and those which do not. This can be restated as follows.

Remark 5. A structure is imposed on each member of $\mathscr{G}$ by the apparent MSGs of other members of $\mathscr{G}$.

Up to this point, we have not assumed the existence of any particular relation between $\mathscr{N}_{x_{0}}$ and $\mathscr{N}_{x_{1}}$. We will now look at the situation where $\mathscr{N}_{x_{1}}$ can be obtained from $\mathscr{N}_{x_{0}}$ by a uniform triaxial extension superimposed on a rigid body rotation (i.e. $\bar{F}=\alpha \overline{\mathbf{R}}$, for some scalar non-zero $\alpha$ and orthogonal $\overline{\mathbf{R}})$. In this case we will show that $\mathscr{B}_{\kappa_{1}}\left(\mathscr{A}_{\kappa_{0}}\right)=\mathscr{A}_{\kappa_{1}}$ and that $\mathscr{B}_{x_{0}}\left(\mathscr{A}_{x_{1}}\right)=\mathscr{A}_{x_{0}}$. That is, symmetries which are represented by orthogonal transformations in $g_{\alpha_{0}}$ will also be represented by orthogonal transformations in $g_{k_{1}}$ and vice versa. If we are given $\mathscr{A}_{\kappa_{0}}$, then $\mathscr{B}_{\kappa_{1}}\left(\mathscr{A}_{\kappa_{0}}\right)$ is given by

$$
\mathscr{B}_{\alpha_{1}}\left(\mathscr{A}_{x_{0}}\right)=\overline{\mathbf{R}} \mathscr{A}_{x_{0}} \overline{\mathbf{R}}^{T} \text {. }
$$

As $\mathscr{A}_{k_{0}}$ only contains orthogonal transformations, it is obvious from (11) that $\mathscr{B}_{x_{1}}\left(\mathscr{A}_{\mathrm{K}_{0}}\right)$ can only contain orthogonal members, which means $\mathscr{A}_{\kappa_{1}}\left(\mathscr{A}_{\kappa_{0}}\right) \subset \mathscr{A}_{\kappa_{1}}$. Similarly, we will have

$$
\mathscr{B}_{\kappa_{0}}\left(\mathscr{A}_{x_{1}}\right)=\overline{\mathbf{R}}^{T} \mathscr{A}_{\kappa_{1}} \overline{\mathbf{R}} \text {, }
$$

which means that $\mathscr{B}_{\alpha_{0}}\left(\mathscr{A}_{\kappa_{1}}\right)$ only contains orthogonal transformations, and therefore $\mathscr{B}_{\kappa_{0}}\left(\mathscr{A}_{\kappa_{1}}\right) \subset \mathscr{A}_{\kappa_{0}}$. The one-to-one mapping in Noll's rule, the fact that the image of $\mathscr{A}_{\kappa_{0}}$ is contained in $\mathscr{A}_{\kappa_{1}}$, and the fact that the image of $\mathscr{A}_{\kappa_{1}}$ is contained in $\mathscr{A}_{\kappa_{0}}$, lead to the conclusion that

$$
\mathscr{B}_{\kappa_{1}}\left(\mathscr{A}_{\kappa_{0}}\right)=\mathscr{A}_{\kappa_{1}} \text {, }
$$

and that

$$
\mathscr{A}_{\mathrm{k}_{0}}\left(\mathscr{A}_{\mathrm{K}_{1}}\right)=\mathscr{A}_{\mathrm{k}_{0}} \text {. }
$$

Remark 6 summarizes these results. 
Remark 6. If $\mathcal{N}_{x_{t}}$ is obtained from $\mathcal{N}_{x_{0}}$ by a uniform triaxial extension superimposed upon a rigid body rotation, then Noll's rule maps $\mathscr{A}_{\mathrm{k}_{0}}$ onto $\mathscr{A}_{\mathrm{k}_{1}}$ and vice versa. In this case, each member of $\mathscr{A}_{\alpha_{1}}$ is obtained from its corresponding member in $\mathscr{A}_{\alpha_{0}}$ by a pure rotation [see equation (11)]. ${ }^{\dagger}$

\section{ANISOTROPIC TENSORS}

In obtaining the results presented later, we have used the results of Rivlin and Smith [5] on anisotropic tensors. The following is a short presentation on anisotropic tensors. The scope of this presentation is restricted to its particular use in this paper. A more detailed presentation can be found in $[5,6]$.

Smith and Rivlin [5] have shown that for a given group $g$ of orthogonal transformations, there is a set of anisotropic tensors $\alpha_{i_{1}}^{(p)} \ldots i_{m}$, for $p=1,2, \ldots$, with the property

$$
x_{i_{1}}^{(p)} \ldots i_{m}=H_{i_{1} j_{1}} \ldots H_{i_{m} j_{m}} x_{j_{1}}^{(p)} \ldots j_{m} \text {, }
$$

for every $H_{i j} \in g$ (the standard summation convention is assumed on repeated indices). This set forms a basis for the construction of any tensor $G_{i_{1}} \ldots i_{n}$ that has the property

$$
G_{i_{1}} \ldots i_{n}=H_{i_{1} j_{1}} \ldots H_{i_{n} j_{n}} G_{j_{1} \ldots j_{n}} \text {, }
$$

for every $H_{i j} \in g$. That is, every $\mathbf{G}$ which satisfies (16) for all $H_{i j} \in g$ can be written as

$$
\mathbf{G}=b_{1} \boldsymbol{\beta}^{(1)}+\ldots+b_{q} \boldsymbol{\beta}^{(q)} \text {, }
$$

for some scalars $b_{1}, \ldots, b_{q}$ and tensors $\boldsymbol{\beta}^{(1)}, \ldots, \boldsymbol{\beta}^{(q)}$ where each tensor $\boldsymbol{\beta}^{(i)}$ is either an anisotropic tensor of $g$ or the tensor product of anisotropic tensors of $g$. This implies that any $G$ which can be written in the form (17) will satisfy (16) and vice versa.

As we will only be concerned with the case where $G$ is a second-rank tensor, equation (16) can be written as

$$
\mathbf{G}=\mathbf{H G H} \mathbf{H}^{T} \text {. }
$$

We will confine the rest of this presentation to such a $\mathbf{G}$.

It is simple to show the following. First, each $\beta^{(i)}$ in (17) commutes with the members of $g$. Second, if we are given a $\mathbf{G}$ which can be written in terms of the anisotropic tensors of either group $g$ or group $g^{*}$, then we are assured that (18) holds for at least every $\mathbf{H} \in g \cup g^{*}$. Third, if $\mathbf{G}$ can be written in terms of the sum of the anisotropic tensors of $g$ plus those of $g^{*}$, then we are assured that (18) holds for at least every $H \in g \cap g^{*}$.

Finally, for those cases where $g$ is the orthogonal generator associated with either transverse isotropy or orthotropy (see below), equation (18) holds for only $\mathbf{H} \in g$ if the following conditions exist:

(1) $\boldsymbol{\beta}^{(1)}, \ldots, \boldsymbol{\beta}^{(q)}$ in (17) include every possible second-rank tensor which is either an anisotropic tensor of $g$ or the outer product of anisotropic tensors of $g$.

(2) No $b_{i}$ in (17) is equal to zero.

(3) No two $b_{i}$ in (17) are equal.

(4) We are only looking for orthogonal $\mathbf{H}$.

The fourth constraint, $\mathbf{H} \in \mathcal{O}$, is not restrictive for our work, but implies that there might exist non-orthogonal $\mathrm{H}$ which satisfy (18).

What follows is a presentation of triclinic, monoclinic, orthotropic, and transversely isotropic materials. For each case we first define an orthogonal generator and then provide the anisotropic tensors associated with this generator. As we only consider those $\mathbf{G}$ which are second-order symmetric tensors in a three-dimensional space, we will present only the anisotropic tensors relevant to this case (see [5] for more details).

\subsection{Triclinic material}

A material is considered triclinic-pinacoidal if its group of MSGs can be generated from

$$
D_{\mathrm{tri}}= \pm\{\mathbf{I}\}
$$

\footnotetext{
This remark is identical to Theorem 2 on p. 200 of [4].

t This would be important if we had not restricted the types of MS under consideration to those which can be generated from a generator which only contains orthogonal members.
} 
where $I$ is the identity. That is, the material is only indifferent to a central inversion. There are nine anisotropic tensors associated with $D_{\text {tri }}$ and they are given as

$$
x_{i j}^{(1)}=\delta_{i 1} \delta_{j 1}, \alpha_{i j}^{(12)}=\delta_{i 1} \delta_{j 2}, \ldots, \alpha_{i j}^{(3)}=\delta_{i 3} \delta_{j 3} \text {, }
$$

where $\delta_{i j}$ is the Kronecker delta. All simple materials which satisfy the constraint of frame indifference are at least triclinic. $D_{\mathrm{tri}}$ is the only member in the group of MSGs of a triclinic material as $\overline{\mathbf{F}} D_{\mathrm{tri}} \overline{\mathbf{F}}^{-1}=\overline{\mathbf{F}}^{-1} D_{\mathrm{tri}}=D_{\mathrm{tri}}$ for every $\overline{\mathbf{F}}$.

\subsection{Monoclinic material}

A material is considered monoclinic-prismatic if its group of MSGs can be generated from

$$
D_{\text {mon }}= \pm\left\{\mathbf{I}, \mathbf{R}_{1}\right\}
$$

where I is the identity, and

$$
\mathbf{R}_{1}=\left(\begin{array}{rrr}
-1 & 0 & 0 \\
0 & 1 & 0 \\
0 & 0 & 1
\end{array}\right)
$$

That is, the material is indifferent to central inversions and reflections about one plane of symmetry (in at least one configuration of the body). There are five anisotropic tensors associated with $D_{\text {mon }}$ and they are given as

$$
a^{(11)}, a^{(22)}, a^{(33)}, a^{(23)}, a^{(32)}
$$

where $a^{(i)}$ is defined in the previous section.

\subsection{Orthotropic (rhombic) material}

A material is considered orthotropic or rhombic-dipyramidal if its group of MSGs can be generated from

$$
D_{\text {orth }}= \pm\left\{\mathbf{I}, \mathbf{R}_{\mathbf{1}}, \mathbf{R}_{\mathbf{2}}, \mathbf{R}_{\mathbf{3}}\right\} \text {, }
$$

where $I$ and $R_{1}$ are the same as given above, and

$$
\mathbf{R}_{2}=\left(\begin{array}{rrr}
1 & 0 & 0 \\
0 & -1 & 0 \\
0 & 0 & 1
\end{array}\right)
$$

and

$$
\mathbf{R}_{3}=\left(\begin{array}{rrr}
1 & 0 & 0 \\
0 & 1 & 0 \\
0 & 0 & -1
\end{array}\right)
$$

That is, the material is indifferent to central inversion and reflections about three perpendicular planes of symmetry (in at least one configuration of the body). There are three anisotropic tensors associated with $D_{\text {orth }}$ and they are given as

$$
a^{(11)}, a^{(22)}, a^{(33)} \text {. }
$$

\subsection{Transversely isotropic material}

A material is considered transversely isotropic if its group of MSGs can be generated from

$$
D_{\text {tran }}= \pm\left\{\mathbf{I}, \mathbf{R}_{1}, \mathbf{M}_{\omega}, \mathbf{R}_{\mathbf{1}} \mathbf{M}_{\omega}, \text { for all } \omega\right\},
$$

where $I$ and $R_{1}$ are given above, and

$$
\mathbf{M}_{\omega}=\left(\begin{array}{ccc}
\cos (\omega) & \sin (\omega) & 0 \\
-\sin (\omega) & \cos (\omega) & 0 \\
0 & 0 & 1
\end{array}\right)
$$


for $0 \leqslant \omega<2 \pi$. That is, the material is indifferent to central inversions, reflections about any plane which passes through a preferred axis of the material, and reflections about a plane perpendicular to the preferred axis of the material (in at least one configuration of the body). There are two anisotropic tensors associated with $D_{\operatorname{tran}}$ and they are given as

$$
a^{(33)}, a^{(11)}+a^{(22)} \text {. }
$$

\section{SYMMETRIES WITH ORTHOGONAL GENERATORS, METHOD FOR FINDING ALL APPARENT MATERIAL SYMMETRY GROUPS}

In the earlier sections, we looked at how the members in a group of MSGs are related, and showed that a particular structure is imposed on each MSG by the apparent MSG of another member. Up to this point, we have been looking at the case of a general MS.

In this section we will restrict our attention to those MS which can be represented, in at least one configuration, by a MSG which contains only orthogonal members. That is, the MS can be represented by an orthogonal generator (one which only contains orthogonal members). The aim of this section is to determine the apparent MSGs of all possible representations of such a MS. We will develop a method for solving for the apparent MSG of one MSG given another MSG and will also find all possible apparent MSGs of an isotropic, transversely isotropic, and orthotropic material. ${ }^{\dagger}$

For the remainder of this section, we will always select $\kappa_{0}$ to be a configuration in which $g_{k_{0}}$ only contains orthogonal members. We will take $g_{x_{0}}$ as the generator of the group of MSGs. The configuration $\kappa_{1}$ will be any arbitrary configuration. $\overline{\mathbf{F}}$ will denote the deformation gradient comparing $\mathscr{N}_{k_{1}}$ to $\mathfrak{H}^{*}$. The polar decomposition of $\overrightarrow{\mathbf{F}}$ will give $\overline{\mathbf{R}}$ for its orthogonal part, $\overline{\mathrm{U}}$ for its right symmetric part, and $\overline{\mathbf{V}}$ for its left symmetric part.

A restatement of our problem can be written as follows.

Problem. Given $g_{\kappa_{0}} \subset \mathcal{O}$, find $\mathscr{A}_{\kappa_{1}}$ for all possible $\overline{\mathbf{F}}$.

The problem will be solved in two parts. First we will find $\mathscr{B}_{x_{0}}\left(\mathscr{O}_{x_{1}}\right)$ and then we will use Noll's rule to find $\mathscr{A}_{\mathrm{x}_{1}}$.

According to Remark 4

$$
\mathscr{B}_{\kappa_{0}}\left(\mathscr{A}_{\kappa_{1}}\right)=g_{\kappa_{0}} \cap D
$$

where

and $\overline{\mathbf{C}}=\overline{\mathbf{F}}^{T} \overline{\mathbf{F}}$.

$$
D=\left\{\mathbf{H} \mid \mathbf{H}^{\top} \tilde{\mathbf{C}} \mathbf{H}=\overline{\mathbf{C}} \quad \text { for } \quad \mathbf{H} \in \mathcal{O}\right\},
$$

As $g_{\kappa_{0}}$ is known, we will proceed to find $D$ for all possible $\overline{\mathbf{F}}$.

To find $D$, we must find all orthogonal $\mathbf{H}$ under which $\bar{C}$ is form-invariant (i.e. $H^{T} \overline{\mathbf{C}} \mathbf{H}$ $=\bar{C}$ ). As $\bar{C}$ is a symmetric positive definite tensor, it can always be written as

$$
\overline{\mathrm{C}}=\mathbf{Q} \overline{\mathrm{C}}_{\mathrm{d}} \mathbf{Q}^{T} \text {, }
$$

for some orthogonal $\mathbf{Q}$ and diagonal $\overline{\mathbf{C}}_{\boldsymbol{d}}$. Let $\overline{\mathbf{C}}_{\mathrm{d}}$ be given by

$$
\left[\overrightarrow{\mathbf{C}}_{\mathrm{d}}\right]=\left(\begin{array}{ccc}
\lambda_{1}^{2} & 0 & 0 \\
0 & \lambda_{2}^{2} & 0 \\
0 & 0 & \lambda_{3}^{2}
\end{array}\right) \text {. }
$$

where $\lambda_{1}^{2}, \lambda_{2}^{2}, \lambda_{3}^{2}$ are the eigenvalues of $\bar{C}$. We can therefore write $\bar{C}_{d}$ as

$$
\overline{\mathrm{C}}_{\mathrm{d}}=\lambda_{1}^{2} a^{(11)}+\lambda_{2}^{2} a^{(22)}+\lambda_{3}^{2} a^{(33)}
$$

where $a^{(i)}$ is defined in (20). As a result of the decomposition (33), we can therefore always write $\overline{\mathbf{C}}$ as

$$
\overline{\mathrm{C}}=\mathrm{Q}\left[\lambda_{1}^{2} a^{(11)}+i_{2}^{2} a^{(22)}+i_{3}^{2} a^{(33)}\right] \mathrm{Q}^{T}
$$

\footnotetext{
To some extent, this work picks up at the point where Theorem 3 on p. 201 of [4] leaves off.
} 
Depending on the eigenvalues of $\overline{\mathbf{C}}$, there will be three distinct cases:

(1) If $i_{1} \neq \lambda_{2} \neq \lambda_{3} \neq \lambda_{1}$ (three distinct eigenvalues), then

$$
\mathbf{H}^{T} \overline{\mathbf{C}}_{\mathrm{d}} \mathrm{H}=\overline{\mathrm{C}}_{\mathrm{d}}
$$

for only $\mathrm{H} \in D_{\text {ortb }}$, and therefore

$$
\mathbf{M}^{T} \overline{\mathbf{C}} \mathbf{M}=\bar{C}
$$

for only $\mathbf{M} \in \mathbf{Q} D_{\text {orth }} \mathbf{Q}^{T}$. This follows directly from (36) and the results presented for the anisotropic tensors of $D_{\text {orth }}$.

(2) If $\lambda_{1}=\lambda_{2} \neq \lambda_{3} \neq \lambda_{1}$ (only two distinct eigenvalues), then (37) holds for only $\mathbf{H} \in D_{\text {tran }}$, and therefore (38) holds for $M \in Q D_{\text {tran }} Q^{T}$. This follows from the fact that $\bar{C}_{d}=i_{1}^{2}\left(a^{(11)}\right)$ $\left.+a^{(22)}\right)+\lambda_{3}^{2} a^{(33)}$ and that $a^{(11)}+a^{(22)}$ and $a^{(33)}$ exhaust all possible second-order tensors which can be obtained from the anisotropic tensors of $D_{\text {tran }}$.

(3) If $i_{1}=\lambda_{2}=\lambda_{3}$, then (37) is true for every $H \in \mathcal{C}$, and therefore (38) is true for every $\mathbf{M} \in \mathbb{C}$.

We will summarize the preceding discussion in the following remark.

Remark 7. Given any arbitrary $\overline{\mathbf{F}}$, such that $\operatorname{det}(\overline{\mathbf{F}}) \neq 0$, depending on the eigenvalues of $\overline{\mathbf{C}}=\overline{\mathbf{F}}^{T} \overline{\mathbf{F}}, D$ will be only one of three distinct cases:

$$
\begin{aligned}
& \text { (a) } D=\mathbf{Q} D_{\text {orth }} \mathbf{Q}^{T} \text { if } \lambda_{1}^{2} \neq \lambda_{2}^{2} \neq \lambda_{3}^{2} \neq i_{1}^{2}, \\
& \text { (b) } D=\mathbf{Q} D_{\text {tran }} \mathbf{Q}^{T} \text { if } i_{1}^{2}=\lambda_{2}^{2} \neq \lambda_{3}^{2}, \\
& \text { (c) } D=\mathcal{O} \text { if } \lambda_{1}^{2}=\lambda_{2}^{2}=\lambda_{3}^{2},
\end{aligned}
$$

for $\lambda_{1}^{2}, \lambda_{2}^{2}$, and $\lambda_{-3}^{2}$ being the eigenvalues of $\bar{C}$ and $Q$ given by $\bar{C}=Q \bar{C}_{d} Q^{T}$ for the diagonal $\bar{C}_{d}$ given in (34).

In the following three sections, we shall find all apparent MSGs of an isotropic, transversely isotropic, and orthotropic material. The procedure in each case would be to find $\mathscr{B}_{\mathrm{x}_{0}}\left(\mathscr{A}_{\mathrm{x}_{1}}\right)$ by intersecting $g_{\mathrm{k}_{0}}$ and the appropriate $D$, from Remark 7 , and then obtain $\mathscr{A}_{\kappa_{1}}$ from $\mathscr{A}_{\kappa_{1}}=\overline{\mathrm{F}} \mathscr{B}_{\kappa_{0}}\left(\mathscr{A}_{\kappa_{1}}\right) \overline{\mathrm{F}}^{-1}$.

\section{ISOTROPIC MATERIAL}

In the case of an isotropic material $g_{\kappa_{0}}=\mathscr{A}_{\kappa_{0}}=\mathcal{C}$. As $\mathscr{B}_{\kappa_{0}}\left(\mathscr{C}_{\kappa_{1}}\right)=g_{\kappa_{0}} \cap D$ and $D \subset \mathbb{C}$, then

$$
\mathscr{B}_{\kappa_{0}}\left(\mathscr{A}_{\kappa_{1}}\right)=D
$$

As $\mathscr{A}_{\kappa_{1}}=\overline{\mathbf{F}} \mathscr{B}_{\kappa_{0}}\left(\mathscr{A}_{\kappa_{1}}\right) \overline{\mathbf{F}}^{-1}$, then

$$
\mathscr{A}_{\mathrm{x}_{\mathrm{t}}}=\overline{\mathbf{F}} D \overline{\mathrm{F}}^{-1} \text {. }
$$

As $\overline{\mathbf{F}}=\overline{\mathbf{R}} \overline{\mathbf{U}}$ and $\overline{\mathbf{U}}=\mathbf{Q} \overline{\mathbf{U}}_{\mathrm{d}} \mathbf{Q}^{\boldsymbol{T}}$ for a diagonal $\overline{\mathbf{U}}_{\mathrm{d}}$ given by

$$
\left[\overline{\mathbf{U}}_{\mathrm{d}}\right]=\left(\begin{array}{ccc}
\lambda_{1} & 0 & 0 \\
0 & i_{2} & 0 \\
0 & 0 & \lambda_{3}
\end{array}\right) \text {, }
$$

then (40) will be

$$
\mathscr{A}_{\mathrm{K}_{1}}=\overline{\mathbf{R}} \mathbf{Q} \overline{\mathbf{U}}_{\mathrm{d}} \mathrm{Q}^{T} D \mathbf{Q} \overline{\mathbf{U}}_{\mathrm{d}}^{-1} \mathbf{Q}^{T} \overline{\mathbf{R}}^{T} .
$$

Remark 7 identifies the following three distinct cases:

(1) If $\lambda_{1} \neq \lambda_{2} \neq \lambda_{3} \neq \lambda_{1}$ (three distinct eigenvalues). This results in

$$
\mathscr{A}_{\kappa_{\mathrm{t}}}=\overline{\mathbf{R}} \mathbf{Q} \overline{\mathbf{U}}_{\mathrm{d}} \mathbf{Q}^{T} \mathbf{Q} D_{\text {orth }} \mathbf{Q}^{T} \mathbf{Q} \overline{\mathbf{U}}_{\mathrm{d}}^{-1} \mathbf{Q}^{T} \overline{\mathbf{R}}^{T} \text {, }
$$

which gives

$$
\mathscr{A}_{\mathrm{K}_{\mathrm{1}}}=\overline{\mathbf{R}} \mathbf{Q} \overline{\mathrm{U}}_{\mathrm{d}} D_{\text {orth }} \overline{\mathrm{U}}_{\mathrm{d}}^{-1} \mathrm{Q}^{T} \overline{\mathbf{R}}^{T} \text {. }
$$

Since $\overline{\mathrm{U}}_{\mathrm{d}}$ can be written in terms of the anisotropic tensors of $D_{\text {orth }}$ and by (18) the anisotropic tensors commute with the transformations of their associated group, $\overline{\mathbf{U}}_{\mathbb{d}} D_{\text {orth }}$ 
$\overline{\mathrm{U}}_{\mathrm{d}}^{-1}=D_{\text {orth }} \mathrm{U}_{\mathrm{d}} \overline{\mathrm{U}}_{\mathrm{d}}^{-1}=D_{\text {orth }}$. This gives

$$
\mathscr{A}_{x_{1}}=\overline{\mathbf{R}} \mathbf{Q} D_{\text {orth }} \mathbf{Q}^{T} \overline{\mathbf{R}}^{T},
$$

which means that the material will appear to be orthotropic. The preferred axes of orthotropy are parallel to the principal axes of $\overline{\mathbf{B}}$, which results from comparing

$$
\overline{\mathbf{B}}=\overline{\mathbf{F}} \overline{\mathbf{F}}^{T}=\overline{\mathbf{R}} \overline{\mathbf{U}} \overline{\mathbf{U}} \overline{\mathbf{R}}^{T}=\overline{\mathbf{R}} \mathbf{Q} \overline{\mathbf{U}}_{\mathrm{d}} \mathbf{Q}^{T} \mathbf{Q} \overline{\mathbf{U}}_{\mathrm{d}} \mathbf{Q}^{T} \overline{\mathbf{R}}^{T}=\overline{\mathbf{R}} \mathbf{Q} \bar{C}_{\mathrm{d}} \mathbf{Q}^{T} \overline{\mathbf{R}}^{T}
$$

with (45).

(2) If $\lambda_{1}=\lambda_{2} \neq \lambda_{3}$ (only two distinct eigenvalues). Through the same procedure as above, it follows that

$$
\mathscr{A}_{x_{1}}=\overline{\mathbf{R}} \mathbf{Q} D_{\operatorname{tran}} \mathbf{Q}^{T} \overline{\mathbf{R}}^{T} \text {. }
$$

Therefore, the material will appear to be transversely isotropic with the preferred axis of transverse isotropy along the principle axis of $\bar{B}$ associated with the eigenvalue $\lambda_{3}$.

(3) If $\lambda_{1}=\lambda_{2}=\lambda_{3}$. In this case

$$
\mathscr{A}_{\mathrm{k}_{1}}=0
$$

The following remark summarizes these results.

Remark 8. The apparent MSG $\mathscr{A}_{\alpha_{1}}$ of an isotropic material with $g_{\alpha_{0}}=\mathcal{C}$ is given by one of the following:
(a) $\mathscr{A}_{\alpha_{1}}=\mathbf{S} D_{\text {orth }} \mathbf{S}^{T}$ if $i_{1} \neq \lambda_{2} \neq \lambda_{3} \neq i_{1}$,
(b) $\mathscr{A}_{\mathrm{x}_{1}}=\mathrm{S} D_{\mathrm{tran}} \mathrm{S}^{T}$ if $\lambda_{1}=\lambda_{2} \neq \lambda_{3}$,
(c) $\mathscr{A}_{x_{1}}=\mathcal{O}$ if $\lambda_{1}=\lambda_{2}=\lambda_{3}$,

where $\lambda_{1}^{2}, \lambda_{2}^{2}$ and $\lambda_{3}^{2}$ are the eigenvalues of $\overline{\mathbf{B}}=\overline{\mathbf{F}} \overline{\mathbf{F}}^{T}$ and $\mathbf{S}=\overline{\mathbf{R}} \mathbf{Q}$ is an orthogonal linear transformation, and comes from the decomposition $\overline{\mathbf{B}}=\mathbf{S} \overline{\mathbf{C}}_{\mathrm{d}} \mathbf{S}^{T}$.

\section{TRANSVERSELY ISOTROPIC MATERIAL}

In the case of a transversely isotropic material, $g_{\kappa_{0}}=\mathbf{P} D_{\mathrm{tran}} \mathbf{P}^{T}$, for some orthogonal transformation $\mathbf{P}$ which determines the direction of the preferred axis of transverse isotropy of the material. The results of this section are summarized in the following remark. The proof follows the remark and contains some of the details omitted in the statement of the remark.

Remark 9. For a given-but otherwise arbitrary- $\overline{\mathbf{F}}$, the apparent MSG $A_{x_{1}}$ of a transversely isotropic material is given by the transformations associated with one of the following material symmetry types:

(a) transversely isotropic,

(b) orthotropic,

(c) monoclinic,

(d) triclinic.

The choice between these cases depends on

(a) the eigenvalues of $\overline{\mathbf{C}}=\overline{\mathbf{F}}^{T} \overline{\mathbf{F}}$,

(b) the material's preferred directions (given through $\mathbf{P}$ ),

(c) the principle directions of $\overline{\mathbf{B}}=\overline{\mathbf{F}} \overline{\mathbf{F}}^{T}$.

Proof. As in the section for an isotropic material, we can write $\bar{U}=Q \bar{U}_{d} Q^{T}$ for some orthogonal $\mathbf{Q}$ and diagonal $\overline{\mathbf{U}}_{\mathrm{d}}$, and therefore $\overline{\mathbf{C}}=\mathbf{Q} \overline{\mathbf{C}}_{\mathrm{d}} \mathbf{Q}^{T}$. Three cases will arise depending on the three eigenvalues of $\overline{\mathbf{U}}$ given by $\lambda_{1}, \lambda_{2}$, and $\lambda_{3}$.

(1) If $\lambda_{1} \neq \lambda_{2} \neq \lambda_{3} \neq \lambda_{1}$, then

$$
D=\mathbf{Q} D_{\text {orth }} \mathbf{Q}^{T}
$$

As

$$
\mathscr{B}_{\mathrm{\kappa}_{0}}\left(\mathscr{A}_{\kappa_{1}}\right)=g_{\kappa_{0}} \cap D
$$

then

$$
\mathscr{B}_{\kappa_{0}}\left(\mathscr{A}_{\kappa_{1}}\right)=\mathbf{P} D_{\text {tran }} \mathrm{P}^{T} \cap \mathrm{Q} D_{\text {orth }} \mathrm{Q}^{T} .
$$


As

$$
\mathbf{Q}^{T} \mathscr{B}_{\kappa_{0}}\left(\mathscr{A}_{\kappa_{1}}\right) \mathbf{Q}=\mathbf{Q}^{T} \mathbf{P} D_{\text {tran }} \mathbf{P}^{T} \mathbf{Q} \cap D_{\text {orth }}
$$

we will have the following subcases:

(a) In the cases where the preferred axis of transverse isotropy (represented by the term $\mathbf{P} D_{\text {tran }} \mathbf{P}^{T}$ ) becomes aligned with one of the directions of orthotropy (represented by the term $\mathbf{Q} D_{\text {orth }} \mathbf{Q}^{T}$ ) we will have

$$
\mathscr{B}_{\mathrm{x}_{0}}\left(\mathscr{A}_{\mathrm{x}_{\mathrm{l}}}\right)=\mathbf{Q} D_{\text {orth }} \mathbf{Q}^{T}
$$

(b) If the preferred axis of transverse isotropy (represented by the term $P D_{\operatorname{tran}} \mathbf{P}^{T}$ ) is in one of the planes of symmetry associated with the orthotropy (given by the term $\mathbf{Q} D_{\text {orth }} \mathbf{Q}^{T}$ ) but is not along any of the axes of orthotropy, then we will obtain one of the three results

$$
\mathscr{A}_{\mathrm{x}_{0}}\left(\mathscr{A}_{\mathrm{k}_{1}}\right)= \pm \mathbf{Q}\left\{\mathbf{I}, \mathbf{R}_{1}\right\} \mathbf{Q}^{T} \text {, }
$$

or

$$
= \pm \mathbf{Q}\left\{\mathbf{I}, \mathbf{R}_{2}\right\} \mathbf{Q}^{T}
$$

or

$$
= \pm \mathbf{Q}\left\{\mathbf{I}, \mathbf{R}_{3}\right\} \mathbf{Q}^{T} \text {. }
$$

The choice between these three cases is made by the determination of the plane of orthotropy which the preferred axis of transverse isotropy falls within. Each of these resuits represents a reflection about one of the three planes of symmetry associated with the orthotropy (represented by the term $\mathbf{Q} D_{\text {orth }} \mathbf{Q}^{T}$ ).

(c) If neither of the above occurs, then

$$
\mathscr{B}_{\kappa_{0}}\left(\mathscr{A}_{\kappa_{1}}\right)= \pm\{\mathbf{I}\}
$$

In each case above we will have

$$
\begin{aligned}
\mathscr{A}_{\kappa_{1}} & =\overline{\mathbf{F}} \mathscr{B}_{\kappa_{0}}\left(\mathscr{A}_{\kappa_{1}}\right) \overline{\mathbf{F}}^{-1}=\overline{\mathbf{R}} \overline{\mathbf{U}} \mathscr{B}_{\kappa_{0}}\left(\mathscr{A}_{\kappa_{1}}\right) \overline{\mathbf{U}}^{-1} \overline{\mathbf{R}}^{T} \\
& =\overline{\mathbf{R}} \mathbf{Q} \overline{\mathbf{U}}_{d} \mathbf{Q}^{T} \mathscr{B}_{\kappa_{0}}\left(\mathscr{A}_{\kappa_{1}}\right) \mathbf{Q} \overline{\mathbf{U}}_{d}^{-1} \mathbf{Q}^{T} \overline{\mathbf{R}}^{T} .
\end{aligned}
$$

As, in each case, $\mathbf{Q}^{T} \mathscr{P}_{\kappa_{0}}\left(\mathscr{A}_{\kappa_{1}}\right) \mathbf{Q}$ is a subset of $D_{\text {orth }}, \overline{\mathrm{U}}_{\mathrm{d}}$ is describable in terms of the anisotropic tensors of $D_{\text {orth }}$, and the anisotropic tensors of $D_{\text {orth }}$ commute with the members of $D_{\text {orth }}$, we will have the apparent material symmetry group of the material given as:

(a) orthotropy along the principle direction of $\overline{\mathbf{B}}=\overline{\mathbf{R}}_{\mathbf{Q}} \overline{\mathbf{B}}_{\mathrm{d}} \mathbf{Q}^{T} \overline{\mathbf{R}}^{T}$ given by

$$
\mathscr{A}_{\kappa_{1}}=\overline{\mathbf{R}} \mathbf{Q} D_{\text {orth }} \mathbf{Q}^{T} \overline{\mathbf{R}}^{T}
$$

(b) a reflection about a principle plane of $\overline{\mathbf{B}}=\overline{\mathbf{R}} \mathbf{Q}_{\mathrm{d}} \mathbf{Q}^{T} \overline{\mathbf{R}}^{T}$ given by

$$
\mathscr{A}_{\kappa_{1}}= \pm \overline{\mathbf{R}} \mathbf{Q}\left\{\mathbf{I}, \mathbf{R}_{1}\right\} \mathbf{Q}^{T} \overline{\mathbf{R}}^{T} \text {, }
$$

or

$$
= \pm \overline{\mathbf{R}} \mathbf{Q}\left\{\mathbf{I}, \mathbf{R}_{2}\right\} \mathbf{Q}^{T} \overline{\mathbf{R}}^{T}
$$

or

$$
= \pm \overline{\mathbf{R}} \mathbf{Q}\left\{\mathbf{I}, \mathbf{R}_{\mathbf{3}}\right\} \mathbf{Q}^{T} \overline{\mathbf{R}}^{T}
$$

(c) or a lack of any symmetry given by

$$
\mathscr{A}_{\kappa_{1}}= \pm\{\mathbf{I}\}
$$

(2) If $\lambda_{1}=\lambda_{2} \neq \lambda_{3}$, then

$$
D=\mathbf{Q} D_{\operatorname{tran}} \mathbf{Q}^{T}
$$

and similar to the first part, we will have

$$
\mathscr{B}_{\kappa_{0}}\left(\mathscr{A}_{\kappa_{1}}\right)=\mathbf{P} D_{\text {tran }} \mathbf{P}^{T} \cap \mathbf{Q} D_{\text {tran }} \mathbf{Q}^{T},
$$

which gives

$$
\mathbf{Q}^{T} \mathscr{B}_{\kappa_{0}}\left(\mathscr{A}_{\kappa_{\mathrm{b}}}\right) \mathbf{Q}=\mathbf{Q}^{T} \mathbf{P} D_{\text {iran }} \mathbf{P}^{T} \mathbf{Q} \cap D_{\text {tran }}
$$


and results in the following subcases:

(a) If $\mathbf{Q}^{T} \mathbf{P} \in D_{\text {tran }}$, then $\mathbf{Q}^{T} \mathbf{P} D_{\text {tran }} \mathbf{P}^{T} \mathbf{Q}=D_{\text {tran }}$ and

$$
B_{\kappa_{0}}\left(\mathcal{A}_{\kappa_{1}}\right)=\mathbf{Q} D_{\operatorname{tran}} \mathbf{Q}^{T} \text {, }
$$

which corresponds to the alignment of the preferred axes of transverse isotropy associated with the two terms in equation (66).

(b) If the two preferred axes of transverse isotropy (associated with the two terms $\mathbf{Q}^{T} \mathbf{P} D_{\text {tran }} \mathbf{P}^{T} \mathbf{Q}$ and $\left.D_{\text {tran }}\right)$ are perpendicular, then

$$
\mathscr{B}_{\kappa_{0}}\left(\mathscr{A}_{\kappa_{2}}\right)=\mathbf{Q} \mathbf{M}_{\phi} D_{\text {orth }} \mathbf{M}_{\phi}^{T} \mathbf{Q}^{T}
$$

for some angle $\phi$ and $\mathbf{M}_{\phi}$ given as

$$
\mathbf{M}_{\phi}=\left(\begin{array}{ccc}
\cos (\phi) & \sin (\phi) & 0 \\
-\sin (\phi) & \cos (\phi) & 0 \\
0 & 0 & 1
\end{array}\right) \text {. }
$$

(c) If neither of the above is true, then

$$
\mathscr{B}_{\kappa_{0}}\left(\mathscr{A}_{\kappa_{1}}\right)= \pm \mathbf{Q} \mathbf{M}_{\phi}\left\{\mathbf{I}, \mathbf{R}_{1}\right\} \mathbf{M}_{\phi}^{T} \mathbf{Q}
$$

for some angle $\phi$ and $\mathbf{M}_{\phi}$ given by equation (70). This last case corresponds to a reflection about the plane passing through both axes of transverse isotropy (associated with the two terms $\mathbf{P} D_{\text {tran }} \mathbf{P}^{T}$ and $\mathbf{Q} D_{\text {tran }} \mathbf{Q}^{T}$ respectively).

In each subcase above, as in equation (59) of the first part, we will have

$$
\mathscr{A}_{\kappa_{1}}=\overline{\mathbf{R}} \mathbf{Q} \overline{\mathbf{U}}_{\mathrm{d}} \mathbf{Q}^{T} \mathscr{B}_{\kappa_{0}}\left(\mathscr{L}_{\kappa_{1}}\right) \mathbf{Q} \overline{\mathbf{U}}_{\mathrm{d}}^{-1} \mathbf{Q}^{T} \overline{\mathbf{R}}^{T} \text {. }
$$

As, for each one of the three cases above, $Q^{T} \mathscr{B}_{\kappa_{0}}\left(\mathscr{A}_{\kappa_{1}}\right) \mathbf{Q}$ is a subset of $D_{\text {tran }}$ and $\bar{U}_{d}$ is constructed from the anisotropic tensors of $D_{\text {tran }}$, we will have the apparent material symmetry group of the material given as

(a) transverse isotropy with preferred direction along one of the principle directions of $\overline{\mathbf{B}}$ $=\overline{\mathbf{R}} \mathbf{Q} \overline{\mathbf{B}}_{\mathrm{d}} \mathbf{Q}^{T} \overline{\mathbf{R}}^{T}$ given by

$$
\mathscr{A}_{x_{1}}=\overline{\mathbf{R}} \mathbf{Q} D_{\operatorname{tran}} \mathbf{Q}^{T} \overline{\mathbf{R}}^{T},
$$

(b) orthotropy along one of the principle axes of

$$
\overline{\mathbf{B}}=\overline{\mathbf{R}} \mathbf{Q} \overline{\mathbf{B}}_{\mathrm{d}} \mathbf{Q}^{T} \overline{\mathbf{R}}^{T}=\overline{\mathbf{R}} \mathbf{Q} \mathbf{M}_{\phi} \overline{\mathbf{B}}_{\mathrm{d}} \mathbf{M}_{\phi}^{T} \mathbf{Q}^{T} \overline{\mathbf{R}}^{T}
$$

given by

$$
\mathscr{A}_{\kappa_{1}}=\overline{\mathbf{R}} \mathbf{Q} \mathbf{M}_{\phi} D_{\text {orth }} \mathbf{M}_{\phi}^{T} \mathbf{Q}^{T} \overline{\mathbf{R}}^{T},
$$

(c) or a reflection about one principle plane of

$$
\overline{\mathbf{B}}=\overline{\mathbf{R}} \mathbf{Q} \overline{\mathbf{B}}_{\mathrm{d}} \mathbf{Q}^{T} \overline{\mathbf{R}}^{T}=\overline{\mathbf{R}} \mathbf{Q} \mathbf{M}_{\phi} \overline{\mathbf{B}}_{\mathrm{d}} \mathbf{M}_{\phi}^{T} \cdot \mathbf{Q}^{T} \overline{\mathbf{R}}^{T}
$$

given by

$$
\mathscr{A}_{\kappa_{1}}= \pm \overline{\mathbf{R}} \mathbf{Q} \mathbf{M}_{\phi}\left\{\mathbf{I}, \mathbf{R}_{1}\right\} \mathbf{M}_{\phi}^{T} \mathbf{Q}^{T} \overline{\mathbf{R}}^{T} .
$$

(3) If $\lambda_{1}=\lambda_{2}=\lambda_{3}$, then by Remark 6 we will have

$$
\mathscr{A}_{x_{1}}=\overline{\mathbf{R}} \mathbf{P} D_{\text {tran }} \mathbf{P}^{T} \overline{\mathbf{R}}^{T} \text {, }
$$

which corresponds, by default, to transverse isotropy with preferred direction along one of the preferred directions of $\overrightarrow{\mathbf{B}}=\lambda \mathbf{I}$ and completes the proof.

\section{ORTHOTROPIC MATERIAL}

Using the same notation and procedure as in the last section, for an orthogonal material, we will have $g_{x_{0}}=\mathscr{A}_{x_{0}}=\mathbf{P} D_{o r t h} \mathbf{P}^{T}$ for some orthogonal $\mathrm{P}$ which gives the preferred directions of the material.

Remark 10. For a given-but otherwise arbitrary- $\overline{\mathbf{F}}$, the apparent MSG $\mathscr{A}_{k_{1}}$ of an orthotropic material is given by the transformations associated with one of the following 
material types:

(a) orthotropic,

(b) monoclinic,

(c) or triclinic.

The choice between these cases depends on

(a) the eigenvalues of $\overline{\mathbf{C}}=\overrightarrow{\mathbf{F}}^{\mathrm{T}} \overrightarrow{\mathbf{F}}$,

(b) the material's preferred directions (given through $\mathbf{P}$ ),

(c) the principle directions of $\overline{\mathbf{B}}=\overline{\mathbf{F}} \overline{\mathbf{F}}^{T}$.

Proof. As in the last section we can write $\overline{\mathbf{U}}=\mathbf{Q} \overrightarrow{\mathrm{U}}_{\mathrm{d}} \mathbf{Q}^{T}$ for some orthogonal $\mathbf{Q}$ and diagonal $\overline{\mathbf{U}}_{\mathrm{d}}$, and therefore $\overline{\mathbf{C}}=\mathbf{Q} \bar{C}_{\mathrm{d}} \mathbf{Q}^{T}$. Three cases will arise depending on the three eigenvalues of $\overline{\mathbf{U}}$ given by $\lambda_{1}, \lambda_{2}$, and $\lambda_{3}$ (see Remark 7).

(1) If $i_{1} \neq \lambda_{2} \neq \lambda_{3} \neq \lambda_{1}$, then

$$
D=Q D_{\text {orth }} Q^{T}
$$

and, as

$$
\mathscr{B}_{\kappa_{0}}\left(\mathscr{A}_{\kappa_{1}}\right)=g_{\kappa_{0}} \cap D
$$

then

$$
\mathbf{Q}^{T} \mathscr{B}_{\kappa_{0}}\left(\mathscr{A}_{\kappa_{1}}\right) \mathbf{Q}=\mathbf{Q}^{T} \mathbf{P} D_{\text {orth }} \mathbf{P}^{T} \mathbf{Q} \cap D_{\text {orth }}
$$

Depending on the values of $\mathbf{P}$ and $\mathbf{Q}$ we will obtain one of the three following subcases:

(a) In the case where all three preferred axes of orthotropy (associated with the two terms $\mathbf{P} D_{\text {orth }} \mathbf{P}^{T}$ and $\mathbf{Q} D_{\text {orth }} \mathbf{Q}^{T}$ ) coincide we will have

$$
\mathscr{A}_{x_{0}}\left(\mathscr{A}_{x_{1}}\right)=\mathbf{Q} D_{\text {ortb }} \mathbf{Q}^{T} \text {. }
$$

(b) If only one of the axes of orthotropy (associated with the two terms $\mathbf{Q}^{T} \mathbf{P} D_{\text {ortb }} \mathbf{P}^{T} \mathbf{Q}$ and $D_{\text {orrh }}$ ) coincides, then

$$
\mathscr{B}_{\kappa_{0}}\left(\mathscr{A}_{\kappa_{1}}\right)= \pm \mathbf{Q}\left\{\mathbf{I}, \mathbf{R}_{1}\right\} \mathbf{Q}^{T}
$$

or

$$
= \pm \mathbf{Q}\left\{\mathbf{I}, \mathbf{R}_{2}\right\} \mathbf{Q}^{T}
$$

or

$$
= \pm \mathbf{Q}\left\{\mathbf{I}, \mathbf{R}_{3}\right\} \mathbf{Q}^{T} \text {. }
$$

(c) If none of the axes of orthotropy (associated with the two terms $Q^{T} P D_{\text {orth }} P^{T} Q$ and $D_{\text {orth }}$ ) coincide, then

$$
\mathscr{A}_{\kappa_{0}}\left(\mathscr{A}_{\kappa_{3}}\right)= \pm\{I\}
$$

which corresponds to triclinic symmetry.

For each subcase above, as in the proof of Remark 9, we will have the apparent material symmetry group given by

(a) orthotropy along the principle directions of $\overline{\mathbf{B}}=\overline{\mathbf{R}} \mathbf{Q} \overline{\mathbf{B}}_{\mathrm{d}} \mathbf{Q}^{T} \overline{\mathbf{R}}^{T}$, given by

$$
\mathscr{A}_{\mathrm{k}_{\mathrm{t}}}=\overline{\mathbf{R}} \mathbf{Q} D_{\text {orth }} \mathbf{Q}^{T} \overline{\mathbf{R}}^{T} \text {, }
$$

(b) a reflection about a principle plane of $\overline{\mathbf{B}}=\overline{\mathbf{R}} Q \overline{\mathbf{B}}_{\mathrm{d}} \mathrm{Q}^{T} \overline{\mathbf{R}}^{T}$, given by

$$
\mathscr{A}_{x_{1}}= \pm \tilde{\mathbf{R}} \mathbf{Q}\left\{\mathbf{I}, \mathbf{R}_{1}\right\} \mathbf{Q}^{T} \overline{\mathbf{R}}^{T}
$$

or

$$
= \pm \overline{\mathbf{R}} \mathbf{Q}\left\{\mathbf{I}, \mathbf{R}_{2}\right\} \mathbf{Q}^{T} \overline{\mathbf{R}}^{T}
$$

or

$$
= \pm \overline{\mathbf{R}} \mathbf{Q}\left\{\mathbf{I}, \mathbf{R}_{3}\right\} \mathbf{Q}^{T} \overline{\mathbf{R}}^{T},
$$

(c) or a total lack of symmetry, given by

$$
\mathscr{A}_{\mathrm{x}_{1}}= \pm\{\text { I }\} \text {. }
$$

(2) If $\lambda_{1}=\lambda_{2} \neq \lambda_{3}$, then

$$
D=Q D_{\operatorname{tran}} Q^{T}
$$


and we will have

$$
\mathbf{Q}^{T} \mathscr{B}_{\kappa_{0}}\left(\mathscr{A}_{\kappa_{\mathrm{t}}}\right) \mathbf{Q}=\mathbf{Q}^{T} \mathbf{P} D_{\text {orth }} \mathbf{P}^{T} \mathbf{Q} \cap D_{\text {tran }}
$$

which gives us the three following subcases:

(a) the case where an axis of orthotropy (given by the term $\mathbf{Q}^{T} \mathbf{P} D_{\text {orth }} \mathbf{P}^{T} \mathbf{Q}$ ) coincides with the preferred axis of transverse isotropy (given by the term $D_{\text {tran }}$ ) we will have

$$
\mathscr{P}_{\kappa_{0}}\left(\mathscr{A}_{\kappa_{1}}\right)=\mathbf{P} D_{\text {orth }} \mathbf{P}^{T} \text {. }
$$

(b) If the preferred axis of transverse isotropy (associated with the term $D_{\operatorname{tran}}$ ) is in a plane of orthotropy (associated with the term $\mathbf{Q}^{T} \mathbf{P} D_{\text {ortb }} \mathbf{P}^{T} \mathbf{Q}$ ) but it does not coincide with any of the preferred axes of orthotropy, then

$$
\mathscr{B}_{\kappa_{0}}\left(\mathscr{A}_{\kappa_{1}}\right)= \pm Q M_{\phi}\left\{I, R_{1}\right\} M_{\phi}^{T} Q^{T}
$$

for some angle $\phi$ and $\mathbf{M}_{\phi}$ given by equation (70).

(c) If neither of the two above cases occurs, then there is a lack of symmetry, given by

$$
\mathscr{B}_{x_{0}}\left(\mathscr{A}_{x_{1}}\right)= \pm\{I\} \text {. }
$$

As in the proof of Remark 9, we will have the three cases for the apparent material symmetry group, given as

(a) orthotropy with one of the preferred axes of the material along the principle direction of $\overline{\mathbf{B}}=\overline{\mathbf{R}} \mathbf{Q} \overline{\mathbf{B}}_{\mathrm{d}} \mathbf{Q}^{T} \overline{\mathbf{R}}^{T}$ associated with $\lambda_{3}$ and given by

$$
\mathscr{A}_{x_{\mathrm{t}}}=\overline{\mathbf{R}} \mathbf{P} D_{\text {ortb }} \mathbf{P}^{T} \overline{\mathbf{R}}^{T} \text {, }
$$

(b) a reflection about a principle plane of $\overline{\mathbf{B}}=\overline{\mathbf{R}} \mathbf{Q} \overline{\mathbf{B}}_{\mathrm{d}} \mathbf{Q}^{T} \overline{\mathbf{R}}^{T}$, given by

$$
\mathscr{A}_{\kappa_{1}}= \pm \overline{\mathbf{R}} \mathbf{Q} \mathbf{M}_{\phi}\left\{\mathbf{I}, \mathbf{R}_{1}\right\} \mathbf{M}_{\phi}^{T} \mathbf{Q}^{T} \overline{\mathbf{R}}^{T} \text {, }
$$

(c) or a lack of symmetry, given by

$$
\mathscr{A}_{\mathrm{x}_{1}}= \pm\{1\} \text {. }
$$

(3) If $i_{1}=\lambda_{2}=\lambda_{3}$, then by Remark 6

$$
\mathscr{A}_{k, 1}=\overline{\mathbf{R}} \mathbf{P} D_{\text {orth }} \mathbf{P}^{T} \overline{\mathbf{R}}^{T} \text {, }
$$

which corresponds to a set associated with orthotropy and completes the proof.

\section{SUMMARY}

We started by presenting the basic properties of MSGs. We showed that a MS is represented by a group of MSGs, $\mathscr{G}$, where each member of $\mathscr{G}$ is an explicit representation of the MS; and where every two members of $\mathscr{G}$ are related by Noll's rule. Noll's rule allowed the generation of $\mathscr{G}$ from any of its members. The chosen, but otherwise arbitrary, member was called the generator of $\mathscr{G}$.

We next defined the apparent MSG of a MSG as its subgroup of orthogonal transforma-

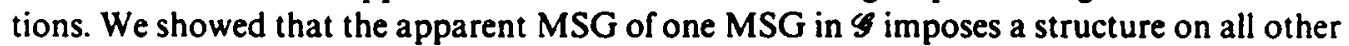
MSGs of $\mathscr{G}$. We used this property to identify all possible apparent MSGs realized in $\mathscr{G}$. Finally, we provided particular results for the cases of an isotropic, orthotropic, and transversely isotropic material.

This work, for the most part, was centered on the identification of apparent MSGs. The importance of this identification was primarily to better understand MS, MSGs, and Noll's rule. In particular, as the orthogonal members of a MSG are the most simply identified members of the MSG, the members of the apparent MSG are the most simply identified members of a MSG.

\section{REFERENCES}

1. W. Noll, A mathematical theory of the mechanical behavior of continuous media. Archs Rational Mech. Anal. 2. 197 (1958). 
2. A.S. Wineman, K. R. Rajagopal and M. Negahban, Change in material symmetry associated with deformation: uniaxial extension. Int. J. engng Sci. 26, 1307 (1988).

3. M. Negahban, Material symmetry and the evolution of anisotropies in first gradient theories of material behavior; inelasticity; polymer crystallization. Dissertation. University of Michigan (1988).

4. C. Truesdell, A First Course in Rational Continuum Mechanics, Vol. 1, General Concepts. Academic Press, New York (1977).

5. G. F. Smith and R. S. Rivlin, The anisotropic tensors. Q. appl. Math. XV, 308 (1957).

6. A. J. M. Spencer, Theory of invariants. In Continuum Physics, Vol. 1, (Edited by A. C. Eringen). Academic Press, New York (1975). 